\title{
Las infancias y el currículo del capital: el caso Divercity
}

\author{
Edisson Cuervo-Montoya, Ph.D. \\ Universidad del Valle, Colombia*
}

Laura María Giraldo-Urrego, Mg.

Universidad de Antioquia, Colombia**

edisson.cuervo@correounivalle.edu.co

\section{Resumen (analítico)}

En Colombia, un modelo de negocio educativo centra su interés en la infancia como nicho de clientes, asegurándose rentabilidad por dos vías: desde el ofrecimiento de servicios de entretenimiento infantil y procesos de formación, y desde la fidelización de marcas comerciales a través de juegos de rol, los cuales pretenden educarles para el «mundo real». El artículo presenta reflexiones teórico-críticas sobre Divercity bajo la estrategia de análisis documental, develando sus intencionalidades y prácticas formativas, así como identificando componentes curriculares que le constituyen desde el punto de vista ideológico-económico. Los resultados apuntan a un creciente ejercicio de consumo instructivo de los infantes en los centros comerciales. Se concluye que existe una consolidación de procesos iniciales de mímesis del consumo adulto y una fidelización de las grandes marcas presentadas por Divercity.

\section{Palabras clave}

Infancia, plan de estudios, consumo, educación, mercado.

\section{Thesauro}

Tesauro de Ciencias Sociales de la Unesco.
Para citar este artículo

Cuervo-Montoya, E., \& Giraldo-Urrego, L. M. (2020). Las infancias y el currículo del capital: el caso Divercity. Revista Latinoamericana de Ciencias Sociales, Niñez y Juventud, 18(3), 1-25. https://dx.doi.org/10.11600/1692715x.18302

\section{Historial}

Recibido: 07.02.2020

Aceptado: 04.03.2020

Publicado: 25.08.2020

\section{Información artículo}

Este artículo forma parte de una línea de investigación consolidada en el seno del grupo de investigación Formaph, en «Estudios curriculares, evaluación y políticas educativas» de la Universidad de Antioquia. El estudio fue realizado entre marzo 7 de 2016 y junio 27 del 2017. Área: Ciencias Sociales. Subárea: Ciencias de la Educación. 


\section{Children and capitalist curriculum: the case of Divercity}

\section{Abstract (analytical)}

In Colombia, an educational business model focuses its interest on children as a niche for clients, ensuring profitability in two ways: offering services that include children's entertainment and educational processes, and, through loyalty to commercial brands, role-playing games that aim to educate them for the «real world». This paper presents theoretical-critical reflections on Divercity using a document analysis strategy, highlighting its intentions and formative practices, identifying curricular components and configuring these using an ideological-economic point of view. The results demonstrate the growing activity of instructive consumption for children in shopping malls. The authors conclude that Divercity consolidates the initial mimesis processes of adult consumption and promotes loyalty to the featured brands..

Keywords

Childhood, curriculum, consumption, education, markets.

\section{Crianças e o currículo do capital: o caso Divercity}

\section{Resumo (analítico)}

Na Colômbia, um modelo de negócios educacional concentra seu interesse na infância como um nicho de clientes, garantindo a rentabilidade de duas maneiras: da oferta de serviços para entretenimento infantil e processos de treinamento e, da lealdade de marcas, através de role-playing games que pretendem educá-los para o «mundo real». O artigo apresenta reflexões teórico-críticas sobre a Divercity, através da estratégia de análise documental, revelando suas intenções e práticas formativas, identificando componentes curriculares que a constituem desde o ponto de vista ideológico-econômico. Os resultados apontam para um crescente exercício de consumo instrucional de crianças em shopping centers. Direcionando as conclusões, para a consolidação dos processos iniciais de mimese do consumo adulto e a lealdade das grandes marcas apresentadas pela Divercity.

\section{Palavras-chave}

Infância, currículo, consumo, educação, mercado.

\section{Información autores}

[*] Profesor Universidad del Valle. Doctor en Ciencias de la Educación de la Universidad de Valencia, España. Magíster en Investigación Educativa de la Universidad de Alicante, España. Licenciado en Filosofía de la Universidad de Antioquia. ID 0000-0001-7793-6825. Índice H5: 3. Correo electrónico:

edisson.cuervo@correounivalle.edu.co

[**] Profesora Universidad de Antioquia. Magíster en Investigación Educativa de la Universidad de Alicante, España. Licenciada en Pedagogía Infantil de la Universidad de Antioquia. (iD) 0000-0002-5167-0114. Índice H5: 1. Correo electrónico: lauram.giraldo@udea.edu.co 


\section{Introducción}

$\mathbf{E}_{\text {y en interrelación con su cultura y contexto (Campos, 2018; Dubar, 20oo; Serra, }}^{1 \text { ser humano construye y reconstruye su identidad desde su condición histórica }}$ 2017). Por esta razón, hoy día puede decirse que la infancia crea y recrea múltiples identidades a partir del influjo social al que es expuesta en la ciudad que habita, por vía de los elementos o dispositivos formativos (o deformativos) instalados en ella; sobre todo si se tiene presente que la ciudad convierte y reconvierte a los niños y niñas en determinados sujetos, por cuanto ella misma se transforma con el pasar del tiempo (Martínez, 2010). La ciudad es dadora de significados con los cuales se interpretan la realidad y la cotidianidad, tal como se plantea en valiosos trabajos de investigación, entre los que cabe citar Ciudades educadoras (Figueras, 2007; Louro, 2019), La ciudad en el curriculum y el curriculum en la ciudad (Martínez, 2010) y Educación y ciudad (Álvarez, 2010).

Y es en dicha ciudad - educadora o deformadora-donde los centros comerciales se han tomado la tarea de coadyuvar en la educación de la infancia. Para el caso colombiano esto no podría ser de otra manera, toda vez que en algunos centros comerciales se han destinado espacios de considerables proporciones para llevar a cabo una tarea educativa no formal. Hablamos de centros recreativos que no solo propician el juego en artefactos mecánicos, sino que también logran lo que para ellos es una particular educación para la vida mediante otros recursos educativos y curriculares. Verbigracia, el caso que observaremos críticamente en el presente artículo: Divercity, una «ciudad (divertida) a escala, donde los niños y las niñas entre los 3 y los 13 años pueden asumir más de 45 roles entre oficios y profesiones mientras aprenden cómo funciona el mundo real» (Divercity, 2018a).

Hay que tener presente que la ciudad, los centros comerciales y nuestro caso de análisis particular (Divercity) son espacios que forman parte de la vida de las personas y, como tales, no son neutros ni carentes de significado, máxime cuando estos mismos encuadran, legitiman y promulgan ideales de ser humano que asientan las bases de un sistema social y político (Acaso \& Nuere, 2005; Martínez, 2010). En otras palabras, dichos 
lugares se encuentran mediados por un currículo de ciudad (Martínez, 2010), a veces explícito y, en la mayoría de las ocasiones, oculto (Jackson, 1968) o proscrito (Cuervo, 2017). Lo anterior, especialmente cuando los contenidos que lo configuran están relacionados con el mercadeo y con la industria del entretenimiento, toda vez que insertan estereotipos, representaciones y conductas que coadyuvan a la perpetuación de paradigmas o formas particulares de comprensión del mundo (Acaso \& Nuere, 2005), en una relación non sancta que puede propiciar particulares intereses comerciales en la población en general y un específico interés en la infancia.

De aquí la importancia de develar y analizar las intenciones de los mensajes implícitos transmitidos por estos espacios de consumo, en cuanto, como refieren Ahwee et al. (2004) en sus tesis, «los curricula [sic] ocultos y nulos tienen como función principal la comunicación y reproducción de los valores de una sociedad. Estos curricula [sic] pueden ser sutiles, insidiosos y perjudiciales, o pueden ser positivos, productivos y justos» (p. 41); aspecto tácito del conocimiento que igual entraña implícitas «actitudes y valores (...), estereotipos y (...) prejuicios» (Ureña \& Mata, 2018, p. 118).

Es relevante traer acá el concepto de eduentretenimiento (del inglés edutainment), neologismo definido como «el proceso de diseñar e implementar una forma mediada de comunicación con el potencial de entretener y educar a las personas, con el objetivo de mejorar y facilitar las diferentes etapas del cambio prosocial (de comportamiento)» (Bouman, 1999, p. 25). Este concepto, si bien puede apuntalar métodos educativos que refuercen o desarrollen procesos socialmente responsables, también puede estar destinado a reforzar o inocular conductas consumistas del pleno neoliberalismo económico (Gaviria \& Echeverry, 1994; Oro, 2018), mediante el conjunto de contenidos implícitos (ocultos) que ofrecen los centros comerciales, en especial el negocio del eduentretenimiento.

En consonancia con lo anterior, se pretenden develar en este texto las implicaciones subjetivas y colectivas que pueden tener dichos espacios y sus prácticas en la infancia, a propósito de los contenidos no declarados en el currículo del capitalismo, particularmente la observación histórica de la infancia. Esta permite entender que, lejos de ser competencia exclusiva del sistema educativo formal e institucional, la infancia es un estado del ser humano sobre el que se tienen influencias sociales, económicas y políticas que, a su vez, están en constante variación y dan cuenta de una convergencia de intereses comerciales y estrategias eduformativas capitalistas de diversa índole; ello que conduce nuevamente a interrogantes como desde quién y para qué se está educando hoy a la infancia, y a través de qué medios. 
Por supuesto que se requiere hacer un breve recorrido sobre el trasfondo de los centros comerciales y los fines sociales que los convocan, más allá de su rol operante en la transformación social de la trama urbana (Medina, 1998). Así mismo, es clave aludir a las «nuevas infancias» que coexisten en la actualidad, una de las cuales - muy centrada en el cosmopolita consumista-dará pie para analizar críticamente su relación con los centros comerciales y, específicamente, con el negocio de eduentretenimiento Divercity. En todo lo anterior es fundamental el currículo de ciudad (Martínez, 2010) como aspecto analítico transversal, ya que, en tanto proporciona directa o indirectamente bases que justifican el proyecto educativo de una cultura (Sacristán \& Pérez, 1983), este se encuentra íntimamente relacionado con el consumo, la infancia y los contenidos del mismo eduentretenimiento (Schor, 2006; Tovar, 2019).

Por tanto, como objetivo principal del presente artículo tenemos el análisis y la revelación de las posibles intencionalidades y prácticas formativas en los parques de Divercity, identificando en ellos los componentes curriculares que los constituyen y los configuran desde el punto de vista ideológico-económico. A partir de lo anterior, surge la siguiente pregunta: ¿cuáles son los procesos de subjetivación que se pueden desprender de una infancia que actualmente habita de manera regular los centros comerciales y, en particular, que asiste a los parques de diversiones Divercity?

\section{Método}

El artículo se deriva de una investigación que se apoya en el paradigma sociocrítico (Denzin \& Lincoln, 2012) y en un diseño de tipo estudio de caso (Simons, 2011; Stake, 1995), para el cual se emplearon técnicas de análisis documental (Galeano, 2012), análisis de información visual (Banks, 2010) y la experimentación práctica del currículum flâneur (Martínez, 2010) como método alternativo de exploración sistemática de la ciudad en cuanto a sus componentes formativos. Así mismo, se contemplaron diferentes fuentes de información (en línea) del parque recreativo y comercial Divercity, al igual que artículos de investigación rastreados de bases de datos reconocidas como Web of Science, ProQuest y Dialnet.

Se analizaron un total de 157 videos compartidos públicamente en YouTube, a partir de una adaptación del análisis del contenido de este tipo de videos propuesto por Arévalo (2017), así como de su sistema categorial para la observación de estos materiales. De igual 
manera, se tuvieron en consideración los trabajos teóricos sobre el análisis del contenido de material visual de Banks (2010), Bardin (1996) y Krippendorff (1997), quienes apuntan al valor de sus significados simbólicos. En ese sentido, las categorías a priori fueron imagen de marcas oficiales (reconocidas en los ámbitos nacional e internacional), imagen de infantes en roles (ejerciendo las profesiones que se ofrecen en Divercity), imagen del adulto (tanto padres de familia como los participantes en cada juego de rol), imagen de infantes con divis (la moneda oficial de Divercity) y lenguaje consumista (expresiones que demarcan un interés materialista). Por su parte, las categorías emergentes fueron contenido testimonial propagandista (infantes y adultos que promocionan Divercity) y justificación pedagógica (empresarios y socios de Divercity que justifican el interés educativo de Divercity).

Por otro lado, se hizo un análisis de la página oficial de la mencionada firma comercial (http://parqueDivercity.com) y se consultaron varios periódicos de circulación nacional (colombiana), diversas revistas, al igual que algunos trabajos académicos de corte crítico y reflexivo, en los que se ha presentado información sobre el mencionado negocio recreativo y «educativo» en particular. Adicionalmente, vale la pena mencionar los análisis de los registros sistemáticos de las observaciones no participantes llevadas a cabo por los investigadores en varias visitas a centros comerciales que albergan sedes de Divercity, así como a otros centros comerciales de diferentes ciudades de Colombia.

Finalmente, la triangulación de la información recabada permitió realizar el análisis y las reflexiones de orden crítico que aquí se exponen, enfatizando las categorías de mayor impacto investigativo reconstruidas gracias a ideas con sentido, a través de las cuales se derivan los resultados. En términos éticos, se debe mencionar que los datos recopilados para el análisis del caso se extractaron en su totalidad de información de acceso abierto y carácter público, tanto en la página de Google Scholar y la página web de Divercity, como en la página de YouTube y demás bases de datos disponibles en la red.

\section{Desarrollo teórico}

En los videos reproducidos se contabilizó, en un principio, el número de apariciones de cada categoría y entre las que tuvieron mayor porcentaje de aparición se destacaron las de imagen de infantes en roles (32\%), imagen de marcas oficiales ( $25 \%$ y lenguaje consumista (21\%). Esto contrastó, a su vez, con las categorías de contenido testimonial propagandista ( $9 \%$, imagen de infantes con divis $(7 \%)$, imagen del adulto $(4 \%)$ y justifi- 
cación pedagógica ( $2 \%$, que fueron las menos recurrentes en la muestra analizada. Así mismo, se llevó un registro temporal de la duración de cada una de estas apariciones, lo que facilitó, a posteriori, iniciar análisis cualitativos detallados que permitieron el surgimiento de las siguientes unidades de sentido.

\section{Consumo y (de)formación capitalista en la infancia: develamiento y análisis}

En razón de lo revisado en la cantidad de datos recopilados, podemos decir que en el marco del objetivo principal de nuestro estudio, en términos de análisis y develamiento de las prácticas formativas que se llevan a cabo en Divercity, se identificaron diversos elementos curriculares que coadyuvan a la materialización de particulares intencionalidades formativas consumistas. Estas están ideológicamente vinculadas con una lectura del mundo de corte capitalista, de la cual se derivan específicos procesos de subjetivación que se encuentran más relacionados con la fidelización a marcas comerciales, prácticas de consumo y roles de sujetos que se desenvuelven en ambientes de oferta y demanda comercial. Es así como se han podido agrupar o reconocer dos grandes categorías, como unidades de análisis: la que hace un reconocimiento de la apuesta «diverconsumista» en Divercity y la nombrada en centros comerciales y procesos de subjetivación infantil capitalista. Veamos:

\section{Una apuesta «diverconsumista»}

Revisado y analizado el material en línea seleccionado como muestra, podemos afirmar que funciona en forma similar a la lógica que opera en los centros comerciales, los cuales precisan servicios nuevos, innovadores y cautivantes que procuren mantener el interés de las personas. En ese orden de ideas, Divercity es una apuesta instructiva comercial —oculta bajo la figura de parque de diversiones- que se sitúa en varios de los centros comerciales de Colombia y de otros países de la región, con miras a lograr un mayor crecimiento en sus formatos fijos y próximamente itinerante, con su nuevo modelo de «Divercity viajero» (Chíquiza, 2018). Divercity se presenta como una ciudad en formato pequeño, adaptada a escala infantil, donde los niños y niñas tienen carta abierta para consumir múltiples bienes, mientras sus padres o cuidadores esperan en la «guardería de padres» (como se llama el sitio de espera de los adultos).

En dicho espacio no hay que preocuparse por hurtos, pérdidas o accidentes que pudieran ocurrirles a los niños y niñas (y que tal vez sucederían por fuera del centro comercial). 
Esto en correspondencia con las tesis críticas de Martínez (2010), cuando se refiere a que las plazas y calles de la ciudad hoy quedan encerradas «en la segura burbuja de un centro comercial (...) invisibilizando algunas de las otras características o elementos de la vida urbana: el conflicto social, el crimen, la violencia o la pobreza» (p. 12). Esto sin mencionar que la infancia callejera, como la denominan Saucedo y Taracena (2011), esa que habita la ciudad desde otras lógicas menos afortunadas, no se topa con el «palacio de cristal» y menos con Divercity.

Divercity es un modelo de negocio innovador que trasciende los formatos tradicionales de los centros comerciales y que ofrece un servicio aparentemente altruista para las familias, ya que, según lo que manifiestan representantes de la empresa en su página oficial, ofrece diversión combinada con «educación»:

Pensando en un nuevo concepto que además de diversión incluya educación, Divercity cuenta con el Mundo Lego Education, un espacio diseñado según la edad de los niños, en el cual pueden desarrollar, mediante procesos de eduentretenimiento, habilidades que ayudan a lograr una formación integral y les permiten desenvolverse social, cultural y laboralmente. (Divercity ahora le apuesta al juego con educación, 2017, §. 2)

Divercity incluye las marcas de empresas del propio centro comercial o de fuera de este, demás de entidades de productos y servicios de la ciudad, compañías, distribuidoras y universidades privadas, entre otros negocios (incluso algunas entidades de orden público). Todo se encuentra ubicado en un espacio destinado solo para niñas y niños, donde habitan una ciudad de ilusiones, exenta de pobreza, peligro, extravíos geográficos, hurtos o secuestros, pero en la que se venden, en cambio, múltiples centros de interés y «sana» diversión consumista para niños, niñas y adultos por separado.

Por ejemplo, grandes marcas como Chevrolet, Toyota, Coca-Cola, Terpel, Tennis, Alpina, Canal Caracol, Teleantioquia, Banco de Bogotá, Avianca, Bodytech, Seguros Bolívar y la Registraduría Nacional se recrean a escala infantil para que los niños y niñas accedan a ellas tal como lo harían los adultos en la ciudad real. De manera análoga, a los niños y niñas se les otorgan tarjetas de crédito y dinero en efectivo de carácter didáctico, que se les entregan en cajeros electrónicos y bancos a escala infantil, con los que interactúan durante su permanencia en dicha ciudad de «sueños».

En razón de la anterior dinámica consumista, Divercity se ha trazado premisas que sustentan su cultura (del capitalismo) en infantes, entre las que se pueden destacar las siguientes: 
En Divercity el que se divierte, gana (...). En Divercity juegas, decides y actúas (...). En Divercity vale lo que eres, lo que sabes, lo que haces (...). En Divercity les das forma a tus ideas (...). En Divercity buscas respuestas a tus preguntas. (Divercity, 2018a)

Premisas culturales que esta empresa complementa y consolida con específicos fines educativos y pedagógicos que coadyuvan para su fundamentación y que, por supuesto, no se dejan al azar. Sus propios constructos argumentativo-pedagógicos (o lo que la misma empresa llama «principios del quehacer educativo»), llevados a cabo dentro de dichas microciudades artificiales (Flores et al., 2017) e infantiles, respaldan cada una de las actividades programadas por Divercity.

Veamos entonces, a manera de ejemplo, algunos de ellos y, a renglón seguido, procedamos a analizarlos críticamente:

En Divercity, los niños sueñan e imaginan: Divercity se centra en la capacidad creativa del niño. La autonomía se desarrolla desde la acción: Divercity es un espacio de acción donde se ejercita la autonomía. Los niños también son ciudadanos: Divercity es una ciudad donde los niños se forman en la ciudadanía. Los niños pueden aprender a ser críticos y activos en las transacciones: en Divercity, los niños aprenden el manejo responsable del dinero. Los niños tienen sus propios medios para aprender: Divercity genera ambientes de aprendizaje significativos. Los niños reflexionan sobre el sentido de la vida: en Divercity, propiciar la reflexión es requisito. (Divercity, 2018a)

De estos principios pedagógicos citados se destacan algunos términos que serán problemáticos y con muy poco sustento educativo. Así, resulta contradictorio afirmar, que Divercity se centra en la capacidad creativa del niño, cuando por el contrario se le trata en ambientes educativos prediseñados que direccionan ideológicamente las decisiones de los propios infantes, en vías de preconcebidas opciones capitalistas de elección. El anterior aspecto choca igualmente con el referido «ejercicio de la autonomía», en la medida en que los niños-niñas-clientes son instruidos, no solo conductual, sino también cognitivamente a través de estos entretenidos juegos de rol. En ellos deben cumplir tareas rentables y económicamente viables para el juego mismo, ya que estos niños y niñas aprenden a ser retribuidos con la respectiva moneda interna del parque (divis). Por otro lado, resulta paradójico asegurar que «los niños pueden aprender a ser críticos» en Divercity, cuando es manifiesto el silenciamiento de la reflexión crítica y autónoma de los sujetos que se ven inmersos en una réplica fidedigna de las condiciones de opresión y 
colonización simbólica que perviven en las sociedades capitalistas y que son evidentes en las sedes de Divercity; prefabricando así ciudadanos alienados ideológicamente en clave capitalista, mientras ellos mismos se divierten jugando el juego del capitalismo.

Recordemos, en relación con lo anterior, que para Acaso y Nuere (2005) los currículos ocultos visualmente (y que operan de manifiesto en Divercity) pretenden perpetuar de manera implícita todo aquello que no resultaría correcto o ético transmitir en forma explícita. Sus reales intenciones no son puestas sobre la mesa y son más bien el resultado de la reproducción de las principales dimensiones de la esfera económica de la sociedad (Torres, 1998), injusta y desigual por demás. Así las cosas, esa «ciudad de los niños», dentro de la estructura y la lógica de los centros comerciales, denota un currículo del capitalismo, en la medida en que tiene un manifiesto ideal de ser humano instrumentalizado bajo ciertos órdenes económicos específicos e individualistas; ello con una marcada estratificación de roles, por cuanto profesiones como la de maestro, conductor o funcionario parece que no existieran; además, se utiliza un lenguaje consumista que incluye sus propias significaciones, en un ambiente de marcas comerciales que se aprehenden de modo permanente en la subjetividad de tales infantes.

\section{Centros comerciales y procesos de subjetivación infantil capitalista}

Divercity opera de manera análoga a las dinámicas del centro comercial. Es un espacio donde al sujeto infante se le provee todo lo necesario para procurar su permanencia allí y que no precise ausentarse de tal «palacio de cristal infantil» para encontrar alimentación, entretenimiento (cines, juegos, servicios sanitarios, ropa, gimnasios, entidades bancarias) y otros almacenes de cadena (Carrefour o Éxito, para el caso de Colombia) gracias a estar incorporados dentro del mismo.

Así, cabe destacar que, si bien en los centros comerciales donde se ubican las sedes de Divercity se admite el ingreso de «cualquier ciudadano», independientemente del estrato socioeconómico, no todos los que acuden al centro comercial pueden ingresar a Divercity. Ello en tanto que, además de reservarse el derecho de admisión, su tarifa actúa como filtro y produce una selección de un público más específico. Por tanto, se puede observar una relación de poder excluyente de estratificación social que, al parecer, es un contenido clave para que lo aprendan los nuevos ciudadanos. No obstante, por lo observado y consultado, la empresa establece precios especiales para instituciones educativas públicas con población infantil vulnerable, como un modo de proyectar clientes que 
podrían garantizar una permanencia de compra y un uso del servicio recibido, gracias al poder de persuasión del currículo oculto que allí opera.

Hablamos de clientes actuales porque debe tenerse en cuenta que, si bien no fue la infancia la que históricamente había consumido, comprado o paseado sola por las calles, plazas o avenidas, hoy día los niños y las niñas han sido identificados como los principales demandantes en el mercado (Buckingham, 2013; Otero \& Giraldo, 2017; Palacio \& Muñoz, 2017). De esta manera, han surgido lugares donde los niños pueden reproducir prácticas adultas de consumo, recibiendo instrucción de compra sin acompañamiento adulto, con el objetivo de que se adiestren autónomamente en el consumo de productos y servicios. Cobra entonces relevancia la existencia de sitios como Divercity, donde los paseos y encuentros tradicionales de antaño se enfrascan para reproducirse de una nueva manera y por medio de nuevos agentes.

Divercity es un espacio de representación que refleja la cultura sociopolítica que lo rodea; es un espejo de los valores culturales que predominan en la sociedad colombiana: el consumo desmedido y el capitalismo extremo. Además, tiene una «idea de desarrollo infantil que se sustenta en una propuesta educativa instrumental, ligada a la lógica neoliberal» (Gómez et. al., 2019, p. 153). Es la apuesta que las industrias y el comercio estaban necesitando. Una experiencia para niños que se asemeja íntimamente a la aparente realidad en pequeño; la cual es buscada, en efecto, por un gran número de personas dentro de la misma sociedad. Y para aquellos niños y niñas cuya realidad socioeconómica dista mucho de ese mundo fantástico de roles adultos, resulta ser un provocador abrebocas a lo que pudiera ser su futuro, su presente o, por lo menos, la proyección de los anhelos sobre productos y servicios concretos.

Según lo revisado en su página web, como por lo encontrado en los documentos estudiados, se debe tener presente que Divercity funciona con ciertos principios pedagógicos que justifican su accionar. Estos promueven una educación para la vida, pero concretamente una vida fundamentada en el consumo, tomando en cuenta las actividades que allí se ejecutan. Es así que quedan desdibujadas ciertas formas de vida, como aquellas que puedan educar quizás para una vida académica, espiritual, solidaria y compasiva (Tobón, 2018), en pro del beneficio de las comunidades, su respectiva ciudad, el país o el mundo en general. Su excusa parece ser siempre que el niño conozca y aprenda sobre el mundo y la vida real por medio de la emulación del mundo adulto a través de roles económicamente aceptados y rentables. 
Sin embargo, en este punto cabe preguntarse qué es lo que imitan aquellos infantes sometidos a los procesos de formación-diversión de Divercity. Este cuestionamiento se resuelve, en términos de Wulf y Gebauer (1995), como un mero ejercicio de mímesis, en el que el niño entra a significarse y representarse por medio de lo que otros dicen que es lo real; y, como se ha expresado, ese mundo real está mediado por lógicas comerciales. Así, dichos procesos de mímesis (que incluso se sedimentan más fuerte que la educación formal) intentan apelar al sujeto capaz de razonar y de criticar.

Estos escenarios de juego, como es el caso de Divercity, comprenden mejor la mímesis que otras instituciones educadoras, por lo que sus efectos serán más rápidos y eficientes al momento de expandir modos de consumo y compra. Resulta incómodo, entonces, educar para la libertad, la autonomía o la voluntad libre cuando el mercado no se sirve de la razón sino de la intensificación de determinados estímulos y afectos para crear adhesiones a marcas que encuadran la propuesta eduformativa y de eduentretenimiento de esta empresa.

La estructuración del sujeto en Divercity se hace en y para el capitalismo y el hiperconsumo. Esto se revela a través del análisis del currículo oculto visual (Acaso \& Nuere, 2005) que se configura en dicha propuesta, denominada «educación para la vida»; actividades del mundo adulto y acciones de la realidad que resultan ser poco más que comercio, consumo y transacciones bancarias. En Divercity, los niños realizan juegos de rol de diferentes profesiones, ligados directamente al dinero y a las tarjetas bancarias, tal como puede observarse en varios de los videos consultados. En estos los niños se disfrazan, asumen el rol de la profesión y tienen «intercambio económico» a través de la moneda interna del parque de diversiones, los llamados divis (Divercity, 2018b). De este modo se corrobora que

aunque no constituya aquí el núcleo central del análisis, no conviene olvidar la práctica colonizadora que determinadas empresas comerciales e instituciones hacen de la idea de la ciudad educadora en términos exclusivamente de marketing, dejando en un alejado segundo plano sus posibles motivaciones educadoras. (Martínez, 2010, pp. 4-5)

Lo interesante en este punto es que el consumo y el marketing sí son para todos (infantes, padres y empresarios) una motivación de tipo educativo, aspecto ya también señalado en la investigación de Soligo et al. (2017).

Espacios como Divercity son sumamente atractivos para todo tipo de población: hombres y mujeres, ricos, clase media y pobres. Esto sigue la misma lógica de los centros 
comerciales, los cuales están construidos de manera tal que mantienen al público en movimiento, observando a su alrededor, atraídos y entretenidos constantemente - pero en ningún caso durante mucho tiempo- por las interminables atracciones que se le ofrecen. No se alienta a detenerse, mirar, conversar, pensar, ponderar y debatir algo distinto de los objetos en exhibición, a pasar el tiempo en actividades desprovistas de valor comercial (Bouman, 1999). Tanto a Divercity como al centro comercial no solo se va a comprar, sino también a pasear; pero, como ya se planteó, son paseos desprovistos de interacción crítica con el otro. Allí el caminante flâneur (Martínez, 2010) pierde todo sentido que no esté mercantilizado. Siguiendo a Martínez (2010), vemos cómo el equipo interdisciplinar del centro comercial (y de Divercity en particular) ha diseñado una réplica del modelo urbano citadino al servicio del consumo, invisibilizando otras propiedades de la vida real: «El conflicto social, el crimen, la violencia o la pobreza» (p. 12).

Puede decirse que hay nuevamente un currículo oculto dentro de sus premisas ya que, por medio de una retórica visión de permanencia, buscan lograr que el consumidor -niño o adulto- prefiera determinadas marcas comerciales por sobre otras que quizás sean de menor costo. Su apuesta por la lealtad (desde infantes) a determinadas marcas resulta interesante, pues un valor que puede construir el ser humano en relación con otro ser humano es traducido y dirigido al comercio explícito, a un objeto inerte, pero que merece el tributo de la lealtad.

Tanto en la página oficial de Divercity Colombia como en el material audiovisual de la empresa se dice que la ciudad y las funciones que allí se enmarcan se ven más reales (Divercity Lima Perú, 2013). Por esto resulta válido preguntarse lo siguiente: ¿qué partes de la ciudad se están reproduciendo?, ¿qué parte de la población se está tomando?, ¿es posible que esta ciudad de marcas y consumo sea la que representa la ciudad más real? Además, una cuestión trascendental: ¿qué clase de sujeto se está formando desde los propósitos educativos o de deformación de un microespacio diseñado para el consumo infantil?

Estas preguntas las responden explícitamente los miembros del grupo comercial de Divercity al afirmar:

El aliado comercial es un actor importante para la ciudad, pues transmite su esencia de marca a los niños y sus familias y hace que la ciudad se vea muy real. Son invitados a participar patrocinando la atracción con la que se identifican. Los niños y sus padres pueden relacionarse con las marcas de la ciudad por medio de una experiencia positiva que genera lealtad y preferencia. (Divercity, 2018a) 
Procesos de subjetivación que en menores de diez años generan identidad, lealtad y preferencia por determinadas marcas; infantes que se presentan completamente expuestos a los vaivenes del mercado, bajo estrategias de juego, mímesis e intervención publicitaria. Este tratamiento directo que coadyuva a configurar la subjetividad de un número muy importante de infantes, tal como lo expresó la gerente de Divercity Bogotá, quien manifiesta que el promedio de visitantes tan solo para el parque de dicha ciudad capitalina fue de 450 ooo personas en el año 2017 y su aspiración para el 2018, a escala nacional, era tener 1000 ooo de visitantes (Chíquiza, 14 de mayo de 2018). Estos infantes son sometidos durante horas a un intencionado adiestramiento psicológico comercial, efectuado con el beneplácito de padres de familia y, en cierta manera, del Ministerio de Educación Nacional de Colombia. Esta institución pierde competencia de intervención y vigilancia en dichos procesos educativos no formales en el ámbito nacional porque esta sociedad empresarial está declarada y registrada como un parque de diversiones y no como lo que es: una entidad de instrucción para el consumo y la formación consumista de clientes-infantes. En virtud de todo lo anterior, la pregunta de si los infantes son un pronóstico de futuro consumista o son precisamente el presente de consumo ya no es tan difícil de responder.

Es necesario señalar que la esfera de formación del sujeto es invadida directamente por imperativos económicos y de consumo que escapan a la capacidad de crítica y evitan un posible distanciamiento de los mencionados espacios por parte del niño. De hecho, escapan incluso al dominio de padres y maestros. Reflejo del avasallamiento sobre la capacidad crítica del sujeto es el caso del artículo académico, derivado de investigaciones de Rosas (2013) y Jurado y Cortés (2018), en el que lejos de pensarse críticamente este espacio, como un lugar donde se propician experiencias de formación consumista, se plantea una visión romántica e idealizada, análoga a los idealistas pasajes de Londres, que vendían la idea de que el centro comercial era el reflejo de la ciudad real. Para Rosas (2013), «la ciudad es un espacio en el que no solo se debe centrar en trabajar y gastar dinero, como no se manifiesta en Divercity, es un espacio para vivir actividades de carácter social, cultural, intelectual y recreativo» (p. 238), conclusión de la que se explica la estrecha relación que establece Divercity con el dinero (o divis) y cada una de sus actividades.

En trabajos como los de Giraldo (2015), Palacio y Muñoz (2017), Runge (2016), Runge et al. (2012) y Runge y Muñoz (2005) se presentan análisis distintos sobre las lecturas del espacio para la infancia, en los que pensar la educación implica necesariamente pensar los espacios donde esta tiene lugar. Sitios diseñados para la regulación y el control de conductas, pero no tanto para el juego desinteresado y la autonomía intelectual. Verbi- 
gracia, el trabajo de Runge et al. (2012), en el que se expone cómo los espacios creados para la infancia no están exentos del gobierno infantil en términos foucaultianos; esto significa que al intervenir en un espacio, se está interviniendo en la población. Intervención, control y modificación de la conducta que se trasladan de los jardines infantiles a los centros comerciales, espacios ambos con visiones de educabilidad efectivas que, per se, no están necesariamente distantes de la neutralidad o filantropía que poseen los espacios para la infancia que Rosas (2013) atribuye a Divercity.

\section{Oferta neoliberal para la niñez}

El niño de hoy es un sujeto de derecho, con voz y voto dentro de la sociedad (por lo menos en la mayoría de los países); por tanto, al menos en teoría, el niño tiene derecho a expresar sus propias opiniones y pensamientos (Unicef, 2006), lo que implica en cierta medida la toma de decisiones. A esto hay que agregarle que, en el fenómeno del consumismo, desde hace ya tiempo el niño ha entrado a cumplir un papel protagónico. Por ejemplo, Buckingham (2013) señala que al menos el niño consumidor tiene una historia desde 1920, año en que el comercio comenzó a concentrarse específicamente en los niños como clientes y muy poco en sus padres. Gracias a su enfoque en el infante, a su poder de elección, a la importancia de sus derechos y necesidades como eje de su adecuado desarrollo, las industrias lo fueron catalogando como un cliente potencial dentro de la adquisición de bienes. En un recuento histórico expuesto por el mismo Buckingham (2013) se indica que «las publicaciones del marketing celebraban la autonomía y agencia de los niños: se representaba al niño consumidor como persona "intencionada, informada y que sabe lo que quiere"» (p. 85).

En relación con lo anterior, se debe hacer hincapié en lo conveniente que resulta para las grandes industrias y superficies comerciales asumir a los niños como agentes de poder de compra y decisión (Gómez et al., 2018), que saben lo que quieren y son críticos, analíticos y exigentes con sus demandas. No obstante, muchos de los que defienden o victimizan a la infancia frente al avasallamiento consumista destacan los perjuicios nefastos que esta intención comercial sobre la infancia les causan al niño en particular y a la sociedad en general, entendiendo que la niñez desempeña más bien un papel pasivo, ya que son inocentes y fáciles de persuadir, cuando ellos mismos se quedan absortos ante las ofertas del medio.

Buckingham (2013) señala que los activistas, al pretender resguardar a la infancia y sus intereses, los presentan a su vez como carentes de poder, al contrario de los comercian- 
tes, quienes aparentemente los manipulan, pero los reconocen como poderosos. Una visión tradicional del niño como inocente (no sabe, no razona), contraria a la del comercio, que destaca y celebra la autonomía y la agencia independiente de los niños.

A partir de ahí puede argüirse que ambas posturas siguen hoy en día coexistiendo y, de cierto modo, polarizando sus visiones. Por ejemplo, en el estudio de Lugo (2013), premiado por la Unicef, y en el de Orofino (2014), se sustenta abiertamente a una infancia mercantilizada; a un niño consumidor con poder de decisión e impacto económico; a un target del mercado donde sus juegos son, a su vez, una estrategia mercantil que lo que menos propende es a una subjetividad infantil crítica.

En otro orden de ideas, dentro de las teorías del juego en la infancia es claro que la emulación del mundo adulto forma parte de los procesos de estructuración del sujeto y sirve de soporte en las representaciones que coadyuvan a la comprensión el mundo. Cuando se es niño se juega a ser astronauta, policía, médico, enfermero, etc., como una aproximación a la inserción en la realidad de la vida adulta. Los juguetes también son parte de este juego simbólico (Piaget \& Inhelder, 1969) y, dado que a lo largo del tiempo (sobre todo con Montessori, 1986) se ha reivindicado su papel educativo y hasta terapéutico, su producción se ha masificado en el ámbito mundial. De lo anterior han sacado partido las industrias del juguete, ya que no solo venden juegos manuales, sino que también promocionan juguetes en los que pueden «estar-entrar»: por ejemplo, carros a escala infantil a los que pueden acceder y manejar, en un intento de emular la acción del adulto. De este modo, la experiencia con el juguete se ha transformado y exige a pasos agigantados una similitud mayor con el objeto y la práctica real; noción que los centros comerciales tienen en cuenta a la hora de involucrar a los niños y las niñas en determinadas atracciones: «Los juguetes realistas e interactivos son la gran apuesta para esta Navidad. Los niños y niñas buscan cada vez más un estímulo en los juguetes. Que hablen, que rían, que lloren... En definitiva, que sean lo más reales posible» (Los juguetes realistas, 2013).

La gran ventaja para la industria y los centros comerciales en la actualidad es que ofrecen todo esto en un espacio «seguro» para niños, niñas, jóvenes y adultos, alejados por supuesto de los asuntos no tan agradables — más bien incómodos- pero reales del mundo adulto e infantil, como las responsabilidades económicas, la pobreza, la mendicidad, la escasez de recursos y la explotación laboral, entre otros aspectos de la vida económica real. Ciertamente, a la industria y al capitalismo no les parece interesante ni estratégico que los niños y niñas contemplen, comprendan e imiten en sus juegos estos fenómenos del mundo de fuera. Lo que muestran a través de los discursos de eduentre- 
tenimiento es más bien un mundo por demás positivo (Chul-Han, 2011), comercial y alejado de la realidad de la existencia humana y social; el cual igualmente está a merced de problemas y contingencias que hacen de la vida misma y de la vida social algo pasado más por el éxito que por el fracaso; más desde el bienestar y no desde los problemas sociales; el amor y menos el desamor. Aunque la realidad sea un conjunto de dichas contradicciones y no solo idealizadas sociedades inexistentes (Chul-Han, 2011).

Ahora bien, es claro que los niños y niñas han crecido - y continúan creciendo-en un mundo cada vez más mercantilizado, saturado de edificios para el consumo, donde todo el comercio tiene algo que ver con ellos. Así, se convierten en el blanco de ofertas y demandas comerciales que se innovan con pluralidad de estrategias cada vez más incisivas en la formación de subjetividades de la infancia. Sin embargo, las tesis de Buckingham (2013) llevan a preguntarse si en esta dinámica cultural los niños y niñas son en realidad víctimas candorosas e inocentes, o más bien son partícipes activos y conscientes de las lógicas del consumo. Sobre esto se preguntaba Giroux (2003) si los infantes son inocentes o culpables del consumo contemporáneo, poniendo así en cuestión la inocencia de los niños y niñas norteamericanos.

Lo cierto es que es que dicha inocencia puede ser un mito cultural, que para algunos sigue teniendo vigencia y es usufructuado por las grandes superficies comerciales. Estas ratifican la inocencia de los infantes, pero, a su vez, los reconocen como agentes autónomos y clientes potenciales de consumo (Bauman, 2012); además, buscan modificar sus tendencias de compra y capacidad de influencia sobre sus padres a la hora de elegir cuál vestido, alimento o juguete es de su predilección para la compra inmediata.

Así pues, la infancia se nos presenta de múltiples formas y no son pocos los estudiosos (Buckingham, 2013; Giroux, 2003; Schor, 2006) que la enfocan como agentes corresponsables de múltiples cambios culturales acontecidos a lo largo del tiempo, entre ellos el del avasallante fenómeno del consumismo. Esta responsabilidad se relaciona con una concepción del niño como conocedor de sus propias acciones y de las consecuencias, contrario a la idea del niño inocente e inmaculado que lo expropia de un saber y, por tanto, de cualquier responsabilidad que impliquen sus acciones. En esta dinámica del consumo está en juego también la formación de una subjetividad que se fragua desde la lógica del consumo (Buckingham, 2013). 


\section{Centros comerciales: ¿en qué forman?}

Un centro comercial puede entenderse como una serie de establecimientos comerciales, independientes pero con criterios de unidad, gestionados por una o varias entidades (Asociación Española de Centros y Parques Comerciales, 2002). Estos, tal como los conocemos en la actualidad, tuvieron sus incursiones iniciales en Estados Unidos en la primera mitad del siglo XX. Esta era una idea comercial no del todo nueva, puesto que su ejecución material estaba inspirada en los pasajes y galerías de la Europa del siglo XIX (Navarro et al., 2005), si bien tales complejos se pueden considerar como producto de la cultura urbana norteamericana (Escudero, 2008). Estos palacios comerciales se expandieron rápidamente por todo el mundo, como lo que Benjamin (1990) rescataba de la guía parisina de 1852: «Pasajes, una nueva invención del lujo industrial» (p. 51). Se presentan como analogías de una ciudad pequeña; espacios donde, en palabras de Cornejo (2007), se compran experiencias de cualquier índole.

Y no debemos olvidar que el mismo Dostoyevski (1864), frente al Palacio de Cristal de Hyde Park, se negó a creer que este fuera la salida para sublimar los vehementes deseos de caos y destrucción que desde siempre han acompañado el trajinar del ser humano. Una idea no muy lejana a la Sloterdijk (2004), quien introduce la metáfora del Palacio de Cristal para aludir a los centros comerciales como producto mismo de la modernidad. Este palacio comercial (la estructura arquitectónica más imponente del siglo XIX) albergaba un espacio dedicado a «la educación del pueblo», la cual apuntaba ya a un capitalismo integral, originando la total absorción del mundo exterior en un interior meticulosamente programado (Vásquez, 2009).

Reconocemos estos espacios prediseñados de comercio o centros comerciales no solo como un lugar de compras o de asueto consumista de infantes y adultos, sino también como lugares de apropiación de contenidos sociales y de formación (o deformación); ello fundamentalmente porque «el centro comercial es el libro de texto de nuestra época. Un espacio en la ciudad visitado por personas diferentes, que aprenden conceptos, procedimientos y actitudes valorativas sobre el mundo (...) del capitalismo espectacular y del hiperconsumo: el reino de la mercancía» (Martínez, 2010, p. 3).

El centro comercial se erige no solo como un lugar de tránsito (para las compras) y la estancia de placer, sino que es un sitio para aprender y aprehender los valores morales que compartimos como sociedad. Es el libro de texto al que millones de niños, niñas, jóvenes y adultos asistimos para conocer cuáles son las últimas innovaciones tecnológicas, cuáles son los sabores que nos identifican desde determinada región (o donde ingresan 
otras regiones del mundo), donde verificamos las tendencias de moda contemporáneas (seña identitaria por excelencia) e, incluso, donde hacemos deporte o asistimos al culto religioso, entre otras actividades sociales que nos enseñan de qué manera debemos vivir en comunidad hoy día. Una suerte de ruina moral que se celebra económicamente sin más.

Ahora bien, en relación con lo dicho hasta este momento, debemos traer a colación la idea de currículo oculto en la ciudad; entre otras cosas porque desde Kemmis (1988) sabemos que el currículo es el resultado de la historia de la humanidad y su realidad social; un medio a través del cual las instituciones, como dispositivos de poder, gobiernan e influencian a la sociedad, con el fin de controlar la producción y reproducción de los procesos educativos (Foucault, 2002). Estos procesos se llevan a cabo actualmente en los centros comerciales, en la medida en que los contenidos comerciales implícitos y publicitarios de tales centros (mercadeo) están mediados por ejercicios de poder, suscitando determinadas experiencias de aprendizaje $-\mathrm{o}$ incluso de instrucción-que se pueden leer en clave curricular (Martínez, 2010). Se trata, entonces, de preguntarnos por las «nuevas funciones» y servicios de «diversión-educación-formación» que estos centros de consumo han incorporado, garantizando con ello la permanencia del público-cliente.

\section{Conclusiones}

Con lo visto y expuesto podemos afirmar, inicialmente sobre infancia y consumo, que los centros comerciales no solo venden artículos, sino también modos de ser y de relacionarse con el mundo. Divercity, como supuesto parque de diversiones desde el punto de vista formal y legal, cumple funciones educativas en la infancia, coadyuva a la formación de identidades y experiencias a partir de las cuales los infantes aprenden a leer el mundo (Giroux, 2003) de manera particularmente capitalista. En ese sentido, Divercity es una nueva tecnología de comercio y consumo que surge con el ánimo de captar la atención de los infantes y colaborarles a los padres con la tarea educativa, debido a que no es suficiente con los juegos y atracciones infantiles que brinda el respectivo centro comercial (carrusel, carros mecánicos infantiles, piscina de pelotas, entre otros), dado que termina siendo más sugerente y atractivo dedicar un espacio exclusivo para que la infancia, como nicho de clientes, consuma de modo independiente, sin ayuda u orientación del adulto. Por ende, se ha creado en este mercado una suerte de subciudad, dentro de la microciudad que ya es propiamente el centro comercial. Allí se brindan alimentación, diversión, servicios sanitarios, oferta de vestuario, un juego de vida adulta traducida 
en juegos de roles mercantiles y, por supuesto, un ejercicio de formación-instrucción para el mercadeo de grandes empresas; esta remarcan en las subjetividades de la población infantil sus propias insignias, generando recordación y aseguramiento de las opciones de compra de estos infantes, que a la vez son futuros adolescentes y adultos que seguirán consumiendo dichas marcas aprendidas en Divercity.

Por todo lo anterior, resulta difícil esperar una ruptura de dicha lógica educativainstruccional a través de este mencionado juego de roles consumistas, cuando para todas las partes (cuidadores, infantes e industria) se genera una suerte de confort educativocomercial; este último nos hace dudar sobre posturas inocentes frente a este currículo del capital que excluye, en cuanto proscribe y anula, cualquier posición crítica del sujeto frente a un divertido mundo infantil de consumo y aprendizaje capitalista. El desarrollo de estas prácticas se facilita por un ejercicio de desujeción de todo posicionamiento crítico-reflexivo, para luego poder sujetarlos a ideales concretos de formación en y para el consumo, saliendo ello fortalecido cuando incluso los padres de familia están cómodos con la oferta de Divercity. Una apuesta diverconsumista en la que, desde la infancia, se procuran la perpetuación y legitimación no solo del consumismo exacerbado de la época, sino también la lealtad y la necesidad de convivir con específicas marcas comerciales que; las cuales, por demás, no son parte de la construcción fantástica del niño, sino que conforman verdaderas alianzas en la industria y el comercio, que circunscribe a los diferentes ciudadanos contemporáneos.

No se pretende concluir poniendo en cuestión si Divercity es o no un espacio educativo, pues evidentemente dicho parque de diversiones tiene unos propósitos y medios que educan; la cuestión es pensar qué tipo de ser humano se está formando a través de tal educación de infantes. En ese sentido, se puede referir que, con lo observado y analizado, este espacio físico, divertido y educativo, deviene una específica forma de estructuración antropológica del sujeto que hace para sí el discurso del capitalismo cotidiano y adulto, implicándose con tales juegos de rol una visión particular de ser humano. Un proyecto de formabilidad que en este caso está (no siempre a manera de currículo oculto) orientado hacia la formación de subjetividades alienadas por la industria y el consumo.

Finalmente, cabe agregar que lo que resulta sugerente en este espacio educativo diverconsumista, y por extensión a los centros comerciales que lo abrigan, no son los juegos de rol adulto que se realizan en él, ni los artículos que se venden en ellos, ni tampoco lo son las entidades bancarias que allí se encuentran para facilitar el acceso al dinero. Lo que resulta problemático en esta dinámica consumista es la lógica totalitaria del sistema, que 
no soporta sujetos por fuera de ella (Mélich, 2006); que no acepta interacciones sociales por fuera de ese palacio de cristal divertido y que ha identificado a la infancia como su ya no tan nuevo objeto de mercado y consumo. A través de ello legitima sus mecanismos de dominación, procurando la consolidación de su modelo pedagógico consumista que se blinda frente otros espacios donde también puede habitar la infancia y configurar su subjetividad de manera solidaria y humanista, y sin una preocupación por el consumo prioritaria.

Como futuras líneas de investigación se plantea la posibilidad de hacer estudios de carácter participativo (a propósito de las narrativas y su relevancia en las ciencias de la educación) que piensan igualmente en espacios de ciudad. Por ello, se precisa conocer percepciones, opiniones o representaciones sociales de los actores que participan en Divercity, aparte de las posturas del profesorado frente a este caso. Se recomienda prestar especial atención a este y otros posibles espacios y programas para la infancia que, revestidos de educación y apuestas pedagógicas modernas, posibilitan la perpetuación de las desigualdades sociales, además de obnubilar la mirada crítica del maestro y tornarla permisiva y conformista con los imperativos capitalistas de la sociedad.

\section{Referencias}

Acaso, M., \& Nuere, S. (2005). El currículum oculto visual: aprender a obedecer a través de la imagen. Arte, Individuo y Sociedad, 17, 205-218.

Ahwee, S., Chiappone, L., Cuevas, P., Galloway, F., Hart, J., Lones, J., Medina, A. L., Menendez, R., Pilonieta, P., Provenzo, E. F., Jr., Shook, A. C., Stephens, P. J., Syrquin, A., \& Tate, B. (2004). The hidden and null curriculums: An experiment in collective educational biography. Educational Studies, 35(1), 25-43.

Álvarez, A. (2010). Educación y ciudad: política pública en la transición pedagógica. Revista Educación y Pedagogía, 22(58), 65-77.

Arévalo, S. (2017, 25-27 de octubre). Propuesta metodológica para el análisis de Youtube y su relación con los movimientos sociales [Ponencia]. II Congreso Internacional Move.net sobre Movimientos Sociales y TIC. https://bit.ly/2VIVPNM

Asociación Española de Centros y Parques Comerciales. (2002). Directorio de los Centros Comerciales de España. Autor.

Banks, M. (2010). Los datos visuales en investigación cualitativa. Morata.

Bardín, L. (1996). Análisis de contenido. Akal. 
Bauman, Z. (2012). Vida de consumo. Fondo de Cultura Económica.

Benjamin, W. (1990). Poesía y capitalismo. Taurus.

Bouman, M. (1999). Collaboration for pro-social change: The turtle and the peacock. The entertainment-education strategy on television [Tesis no publicada]. Wageningen Agricultural University.

Buckingham, D. (2013). La infancia materialista: crecer en la cultura consumista. Morata.

Campos, H. (2018). Estudio de la identidad cultural mediante una construcción epistémica del concepto identidad cultural regional. Cinta de Moebio, (62), 199-212. http://dx.doi.org/10.4067/So717-554X2018000200199

Chíquiza, J. (2018, 14 de mayo). Nuevo concepto de Divercity viajero llegará a Ibagué este año. La República. https://bit.ly/33 NNcVV

Chul-Han, B. (2011). La sociedad del cansancio. Herder.

Cornejo, I. (2007). El lugar de los encuentros: comunicación y cultura en un centro comercial. Universidad Iberoamericana.

Cuervo, E. (2017). Del currículum nulo al «currículum proscrito» o de las formas de segregación de contenidos en la práctica escolar. Revista Notandum, (44/45), 140-151. https://doi.org/10.4025/notandum.44.3

Denzin, N., \& Lincoln, I. (2012). Paradigmas y perspectivas en disputa. Gedisa.

Divercity. (2018a). ¿Quiénes Somos? https://bit.ly/2tYapDt

Divercity. (2018b). Divis, la moneda oficial. https://bit.ly/2NZrDXo

Divercity ahora le apuesta al juego con educación. (2017, 29 de agosto). Extra. https:// bit.ly $/ 2 \mathrm{NXdc5} \mathrm{U}$

Divercity Lima Perú. (2013). ¿Qué es Divercity? [Video]. YouTube. https://bit.ly/2FfPdjB

Dostoyevski, F. (1864). Memorias del subsuelo. Luarna.

Dubar, C. (2000). La socialisation. Armand Colin.

Escudero, L. (2008). Los centros comerciales, espacios posmodernos de ocio y consumo: un estudio geográfico. Ediciones de la Universidad de Castilla-La Mancha.

Figueras, P. (2007). Ciudades educadoras: una apuesta por la educación. Participación Educativa, 6, 22-27.

Flores, P., Gómez, N., \& Osman, J. (2017). Urbanismo e infancia: hacia un modelo de ciudad que promueva la conciencia ambiental. Revista Latinoamericana de Comunicación, 136, 81-96.

Foucault, M. (2002). Vigilar y castigar: nacimiento de la prisión. Siglo XXI.

Galeano, M. E. (2012). Estrategias de investigación social cualitativa: el giro en la mirada. La Carreta. 
Gaviria, M., \& Echeverri. O. (1994). Neoliberalismo: una nueva concepción del liberalismo económico. Páginas. Revista Académica e Institucional de la UCPR, (46), 1-10.

Giraldo, L. (2015). El espacio y su uso en la construcción social de la norma. Zona Próxima, (23), 49-72. https://doi.org/10.14482/zp.23.6035

Giroux, H. (2003). La inocencia robada: juventud, mutilaciones y política cultural. Morata.

Gómez, M., García-Gutiérrez, L., \& García-Guevara, Á. (2019). Crítica a la idea de infancia y proyecto educativo en las políticas públicas de Colombia. Revista Latinoamericana de Ciencias Sociales, Niñez y Juventud, 17(1), 153-168. https://doi.org/ 10.11600/1692715x.17109

Gómez, P., Castro, A., \& Aguadez, J. (2018). La publicidad con ojos de niño: imaginarios infantiles y construcción crítica de significados. Aula Abierta, 47(4), 471-480. https:// doi.org/10.17811/rifie.47·4.2018.471-480

Jackson, P. (1968). Life in classrooms. Holt, Rinehart and Winston.

Jurado, A., \& Cortés, D. A. (2018). El centro comercial como tercer lugar [Tesis de maestría no publicada]. Universidad Externado de Colombia.

Kemmis, S. (1988). El currículum: más allá de la teoría de la reproducción. Morata.

Krippendorff, K. (1997). Metodología de análisis de contenido teoría y práctica. Paidós.

Los juguetes realistas e interactivos son la gran apuesta para esta Navidad. (2013, 6 de noviembre). Reason Why. https://bit.ly/2PNjero

Louro, P. (2019). Uma introdução ao «Cidade Educadora: olhares e práticas». Kultur. Revista Interdisciplinária sobre la Cultura de la Ciutat, 6(11), 17-26. https://doi.org/ 10.6035/Kult-ur.2019.6.11.1

Lugo, G. (2013). Derechos de los niños y espacios jugables: la reconceptualización del juego y el niño como una estrategia para la definición de un nuevo paradigma de espacios públicos abiertos a escala vecinal para la ciudad de Puebla [Tesis doctoral no publicada]. Benemérita Universidad Autónoma de Puebla.

Martínez, J. (2010). La ciudad en el curriculum y el curriculum en la ciudad. Morata.

Medina, F. (1998). El centro comercial: una burbuja de cristal. Estudios sobre las Culturas Contemporáneas, $4(8), 61-91$.

Mélich, J. (2006). Tres ensayos de filosofía de la educación. Miño y Dávila.

Montessori, M. (1986). La mente absorbente del niño. Diana.

Navarro, J., Martí, P., \& Ortuño, A. (2005). Centros comerciales en áreas urbanas de tamaño medio. Ministerio de Vivienda.

Oro, L. (2018). El neoliberalismo como horizonte cultural. La Razón Histórica: Revista Hispanoamericana de Historia de las Ideas Políticas y Sociales, (38), 21-38. 
Orofino, M. (2014). O ponto de vista da criança no debate sobre comunicação e consumo. Revista Latinoamericana de Ciencias Sociales, Niñez y Juventud, 13(1), 369-381.

Otero, M., \& Giraldo, W. (2017). Consumo de productos infantiles en la base de la pirámide poblacional: análisis de los mecanismos influyentes. Económicas CUC, 38(1), 165-184. https://doi.org/10.17981/econcuc.38.1.08

Palacio, L., \& Muñoz, D. A. (2017). Infancia en la sociedad de consumo: subjetivación, control y resistencia ética. En-Clave Social, 6(1), 50-56.

Piaget, J., \& Inhelder, B. (1969). Psicología del niño. Morata.

Rosas, Á. (2013). Vivencia de ciudad en Divercity. Universidad Pontificia Bolivariana.

Runge, A. (2016). De la calle a la casa, de la casa a la habitación y al centro comercial: espacios para la domesticación y gobierno de la infancia contemporánea de Medellín. Revista Cadernos de Educação, (55), 56-77.

Runge, A., Carrillo, S., \& Muñoz, D. (2012). Los «mega» jardines infantiles de Medellín y el gobierno de la población infantil. Itinerario Educativo, 26(60), 55-74. https:// doi.org/10.21500/01212753.1400

Runge, A., \& Muñoz, D. (2005). Mundo de la vida, espacios pedagógicos, espacios escolares y ex-centricidad humana: reflexiones antropológico-pedagógicas y sociofenomenológicas. Revista Latinoamericana de Ciencias Sociales, Niñez y Juventud, $3(2), 2-21$

Sacristán, G., \& Pérez, G. A. (1983). La enseñanza: su teoría y su práctica. Akal.

Saucedo, I., \& Taracena, B. (2011). Habitar la calle: pasos hacia una ciudadanía a partir de este espacio. Revista Latinoamericana de Ciencias Sociales, Niñez y Juventud, 9(1), $269-285$.

Schor, J. (2006). Nacidos para comprar: los nuevos consumidores infantiles. Paidós.

Serra, A. (2017). Mirar el espacio público con ojos de la infancia. Aula de Innovación Educativa, (261), 25-28.

Simons, H. (2011). El estudio de caso: teoría y práctica. Morata.

Sloterdijk, P. (2004). El Palacio de Cristal. Centro de Cultura Contemporánea de Barcelona. Soligo, V., Derisso, J., \& Conceição, L. (2017). Consumismo infantil, indústria cultural e educação escolar. Educere et Educare, $12(25), 1-9$.

Stake, R. (1995). The art of case research. Sage.

Tobón, D. (2018). Cultura consumista y políticas de la compassion. Escritos, 26(56), 151-166.

Torres, J. (1998). El currículum oculto. Morata.

Tovar, M. (2019). Material didáctico audiovisual de edu-entretenimiento y su incorporación en contextos educativos. Revista Mexicana de Bachillerato a Distancia, 11(22), 1-6. https://doi.org/10.22201/cuaed.20074751e.2019.22.70579 
Unicef. (2006). Convención sobre los Derechos del Niño. Unicef.

Ureña, O., \& Mata, A. (2018). ¿Qué sabemos qué se enseña en filosofía?: el currículo oculto de una formación filosófica. Ensayos Pedagógicos, (edición especial), 115-141.

Vásquez, A. (2009). Peter Sloterdijk y Walter Benjamin; Air Conditioning en el mundo interior del capital. Nómadas, 22(2), 25-38.

Wulf, C., \& Gebauer, G. (1995). Mimesis: Culture-Art-Society. University of California Press. 